Supporting information for

\title{
'Atom Probe Tomography Analysis of Boron and/or Phosphorus Distribution in Doped Silicon Nanocrystals'
}

\author{
Keita Nomoto ${ }^{\dagger}$ Hiroshi Sugimoto, ${ }^{,}$Andrew Breen, ${ }^{\S}$ Anna V. Ceguerra, ${ }^{\S}$ Takashi Kanno, ${ }^{+}$ \\ Simon P. Ringer, Ivan Perez Wurfl, ${ }^{\dagger}$ Gavin Conibeer, ${ }^{,+}$Minoru Fujii ${ }^{*},+$ \\ 'School of Photovoltaic and Renewable Energy Engineering, The University of New South \\ Wales, NSW 2052, Australia \\ ${ }^{ \pm}$Department of Electrical and Electronic Engineering, Graduate School of Engineering, Kobe \\ University, Rokkodai, Nada, Kobe 657-8501, Japan \\ ${ }^{\S}$ Australian Centre for Microscopy \& Microanalysis, and School of Aerospace, Mechanical \\ and Mechatronic Engineering, The University of Sydney, NSW 2006, Australia \\ "Australian Institute for Nanoscale Science and Technology, and School of Aerospace, \\ Mechanical and Mechatronic Engineering, The University of Sydney, NSW 2006, Australia \\ Corresponding Authors: \\ *E-mail: g.conibeer@unsw.edu.au. Phone: +61-2-9385-7858. (G. C.) \\ *E-mail: fujii@eedept.kobe-u.ac.jp. Phone:+81-78-803-6081. (M. F.)
}

In this supporting information the following figures are provided.

- $\quad$ Figure S1: Mass spectra of the different samples used in the reconstruction.

- Figure S2: 3D reconstructions of co-doped Si NCs in BPSG annealed at $1050{ }^{\circ} \mathrm{C}$ and $1250{ }^{\circ} \mathrm{C}$.

- $\quad$ Figure S3: Size distribution of solely- and co-doped Si NCs.

- Table S1: Number density of Si NCs in BSG, PSG, and BPSG.

- Figure S4: Proxigram analysis on co-doped Si NCs in BPSG annealed at $1150{ }^{\circ} \mathrm{C}$ for a variety of Si NCs size ranges.

- $\quad$ Figure S5: Individual solely- and co-doped Si NCs with varieties of diameters. 
Figure S1 shows the mass spectra of the different samples used in the reconstruction. All selected ranges are used in the $3 \mathrm{D}$ reconstruction and influences any subsequent chemical analysis.

Figure S2 shows 3D reconstructions of co-doped Si NCs in BPSG annealed at $1050{ }^{\circ} \mathrm{C}$ and $1250{ }^{\circ} \mathrm{C}$. Sliced images (box size: $24 \mathrm{~nm} \times 24 \mathrm{~nm} \times 7 \mathrm{~nm}$ ) are shown below each tip. In the sliced images, the $\mathrm{B}$ and $\mathrm{P}$ enrichment at the position where the $\mathrm{Si}$ density is high is more prominent when the annealing temperature increases from $1050{ }^{\circ} \mathrm{C}$ to $1250{ }^{\circ} \mathrm{C}$.

Figure S3 shows the size distribution of solely- and co-doped Si NCs. The diameter is derived from the volume created by an iso-concentration surfaces bounding volumes that contain at least 57 at. \% Si. Although the total number of Si NCs and the size is widely distributed, there is a clear trend that the mean diameter increases by co-doing or by higher annealing temperature.

Table S1 Assuming the atomic density of the samples ( $\mathrm{SiO}_{1.5}$ in this case) is 30 atom $/ \mathrm{nm}^{3}$, the total volume of the whole tips were derived from the number of total ions. The number density, the number of Si NCs per unit volume, was divided the number of Si NCs (Figure 1 in the main text) by the total volume.

Figure S4 provides proxigram analysis on co-doped Si NCs in BPSG annealed at $1150{ }^{\circ} \mathrm{C}$ for a variety of $\mathrm{Si}$ NCs size ranges. Si NCs size ranges were selected as small NCs $(2 \mathrm{~nm}<\mathrm{d}<3 \mathrm{~nm})$, medium NCs $(3 \mathrm{~nm}<\mathrm{d}<4 \mathrm{~nm})$, and large NCs $(\mathrm{d}<4 \mathrm{~nm})$. It is confirmed that the $\mathrm{B}$ and $\mathrm{P}$ concentration and distribution profiles are almost the same regardless of the Si NCs size ranges.

Figure S5 shows individual solely- and co-doped Si NCs grown at different temperatures with varieties of diameters. The B and/or P incorporation is confirmed for all samples even for small Si NCs below $2.0 \mathrm{~nm}$ in diameter. 

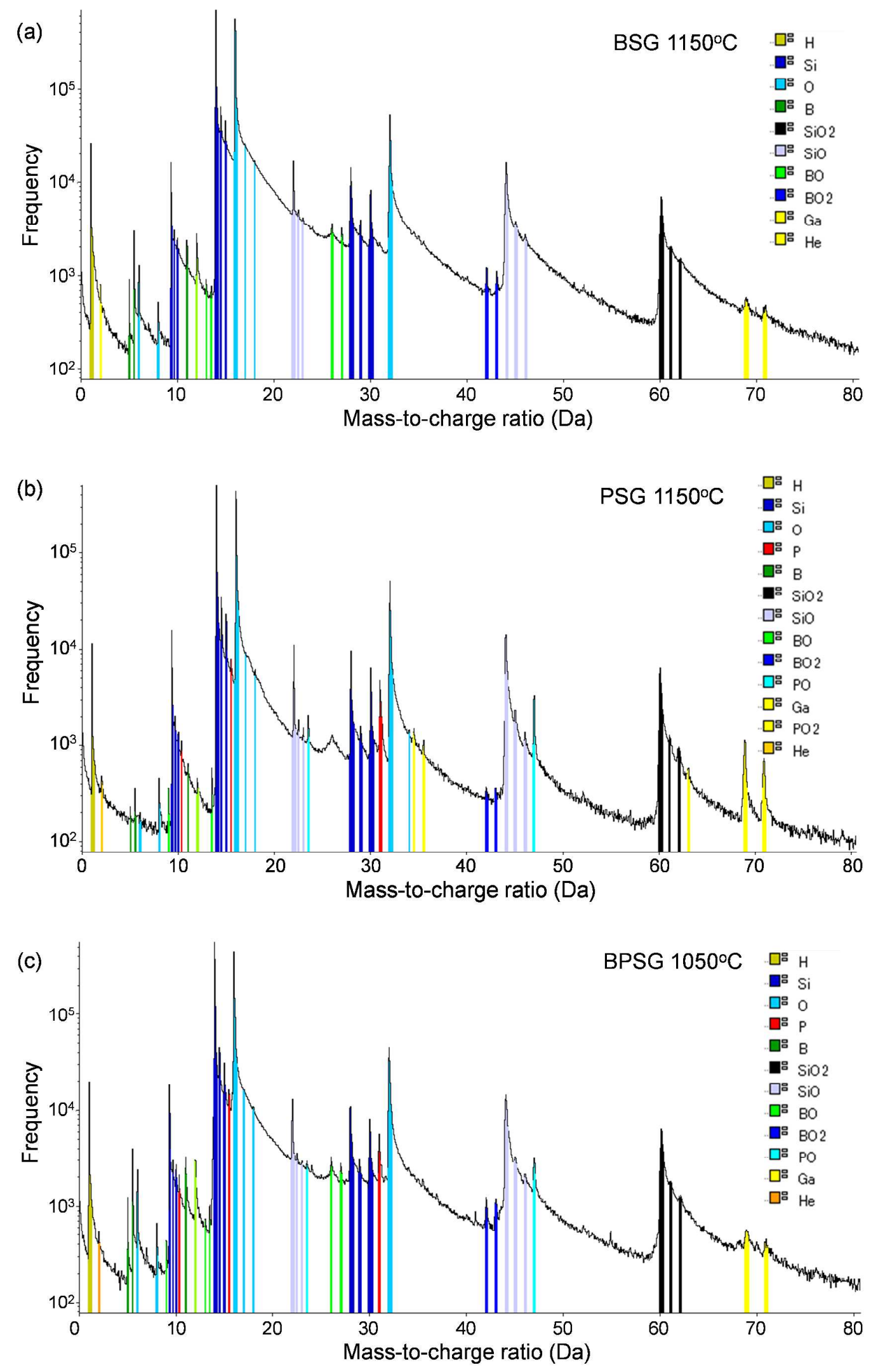

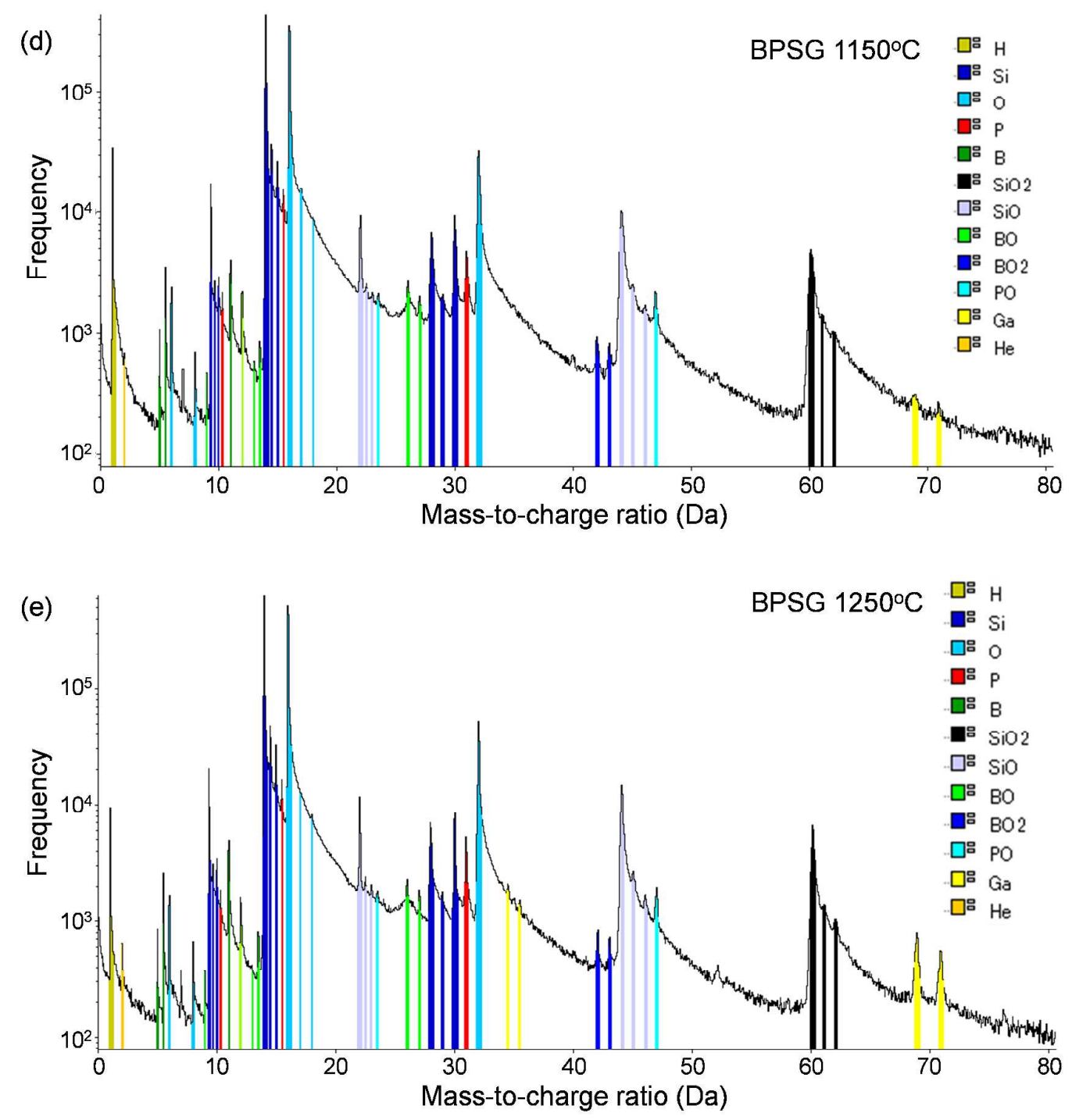

Figure S1. Mass spectra of the different samples used in the reconstruction. (a) BSG annealed at $1150{ }^{\circ} \mathrm{C}$, (b) PSG annealed at $1150{ }^{\circ} \mathrm{C}$, (c) BPSG annealed at $1050{ }^{\circ} \mathrm{C}$. (d) BPSG annealed at $1150{ }^{\circ} \mathrm{C}$, and (e) BPSG annealed at $1250^{\circ} \mathrm{C}$. 
(a) BPSG $1050^{\circ} \mathrm{C}$
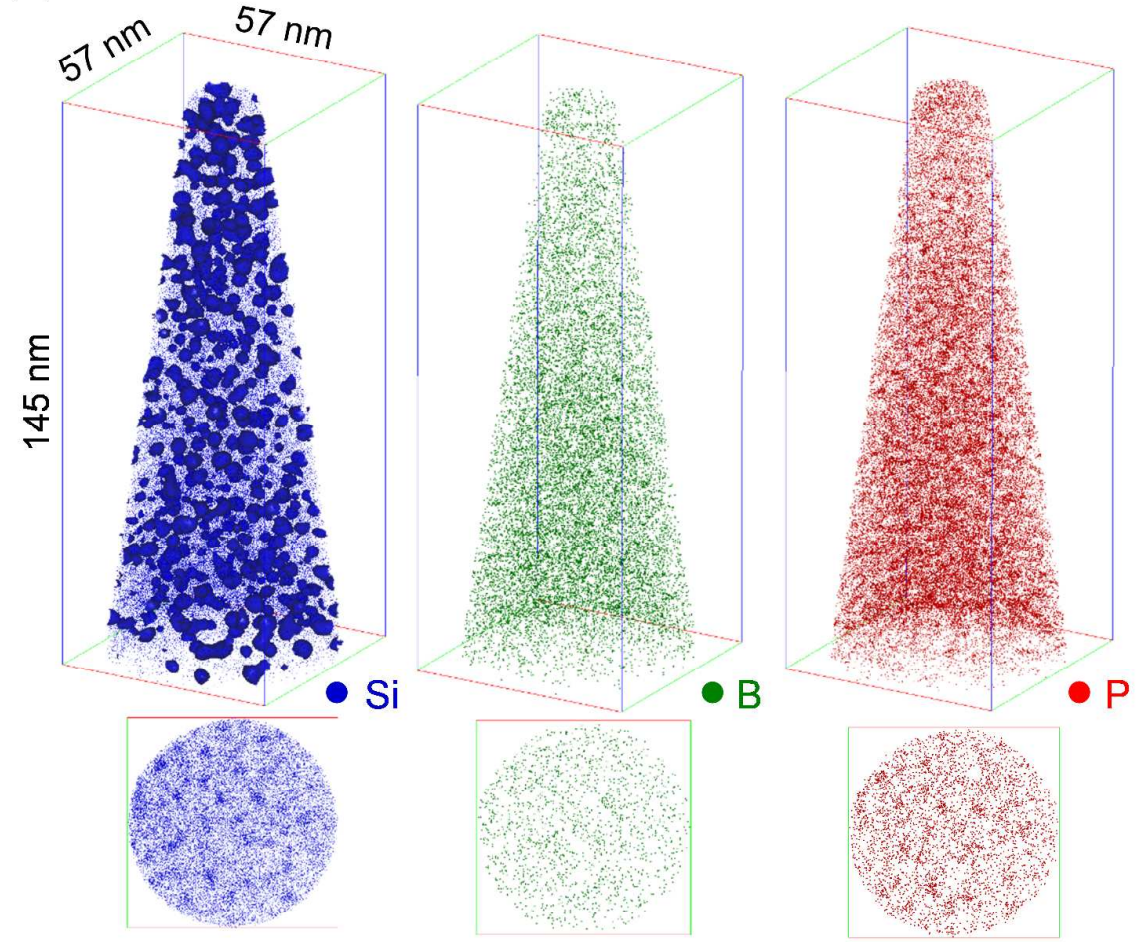

(b) BPSG $1250^{\circ} \mathrm{C}$
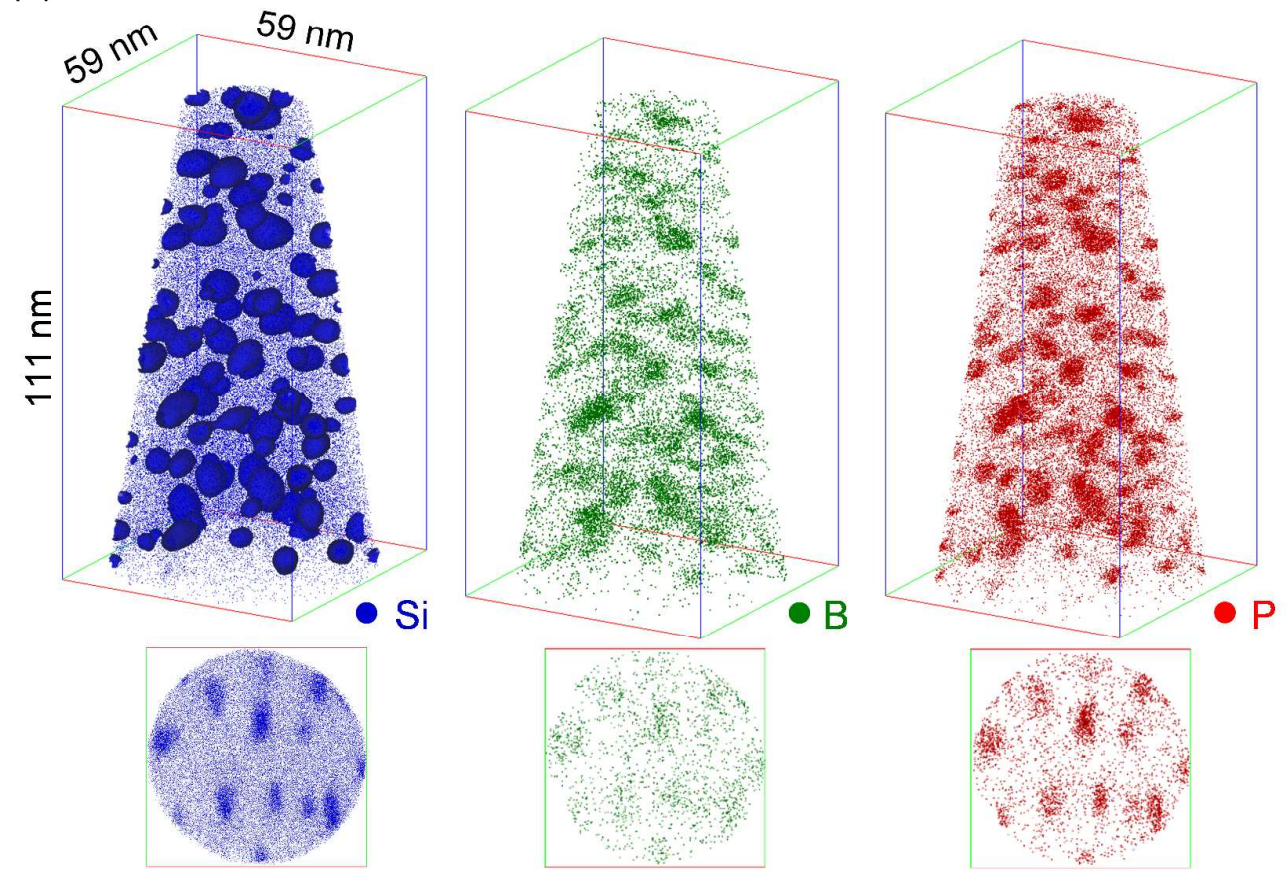

Figure S2. 3D reconstructions of co-doped Si NCs in BPSG annealed at (a) $1050{ }^{\circ} \mathrm{C}$ and (b) $1250{ }^{\circ} \mathrm{C}$. Sliced images (box size: $24 \mathrm{~nm} \times 24 \mathrm{~nm} \times 7 \mathrm{~nm}$ ) are shown below each tip (top view). 

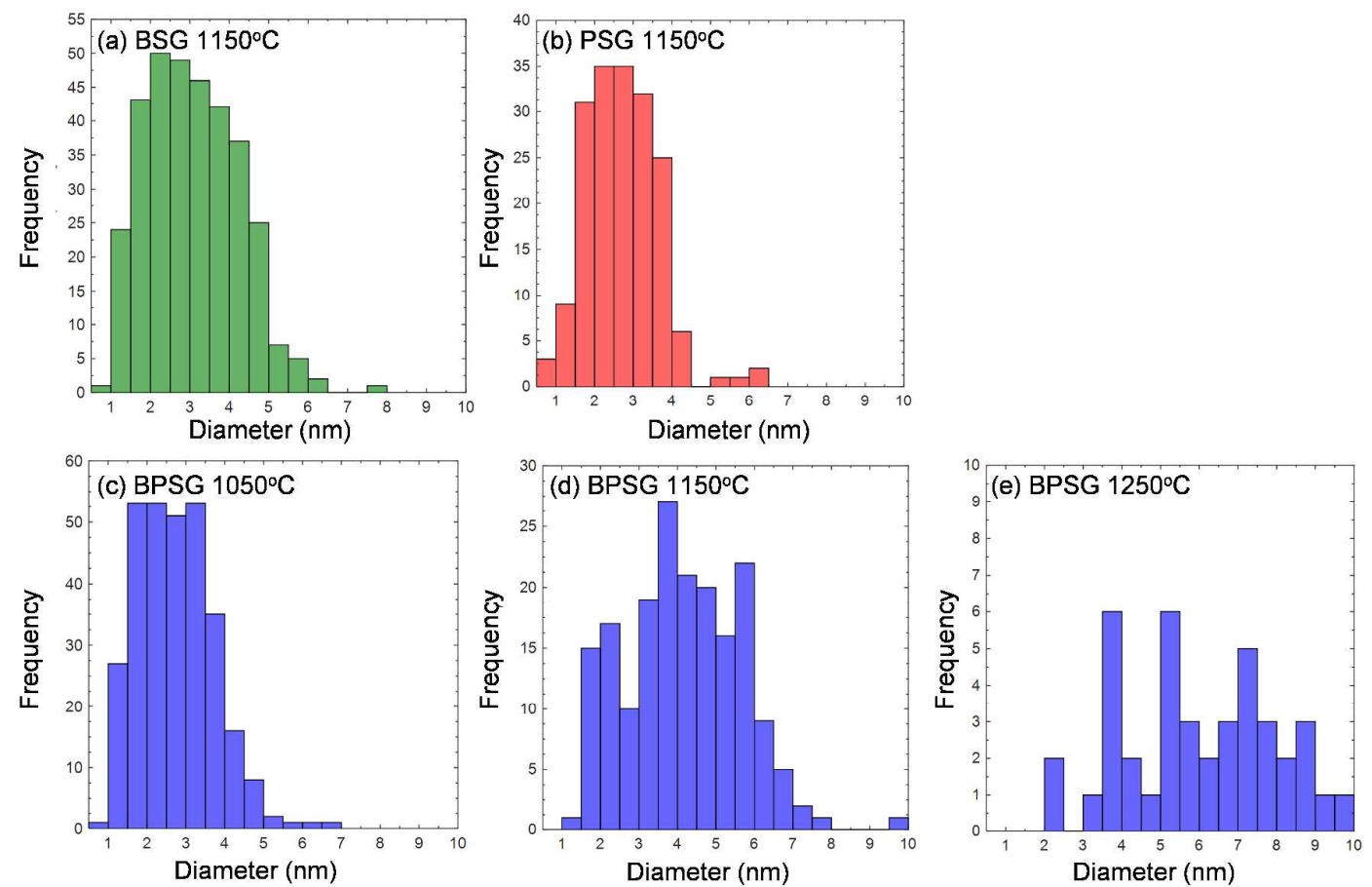

Figure S3. The size distribution of Si NCs in (a) BSG annealed at $1150{ }^{\circ} \mathrm{C}$, (b) PSG annealed at $1150{ }^{\circ} \mathrm{C}$, (c) BPSG annealed at $1050{ }^{\circ} \mathrm{C}$. (d) BPSG annealed at $1150{ }^{\circ} \mathrm{C}$, and (e) BPSG annealed at $1250{ }^{\circ} \mathrm{C}$.

Table S1. Number density of Si NCs in BSG, PSG, and BPSG.

\begin{tabular}{ccc}
\hline \hline Sample & Annealing temperature & Number density $\left(\mathrm{cm}^{-3}\right)$ \\
\hline BSG & $1150^{\circ} \mathrm{C}$ & $2.0 \times 10^{18}$ \\
\hline PSG & $1150^{\circ} \mathrm{C}$ & $1.5 \times 10^{18}$ \\
\hline \multirow{3}{*}{ BPSG } & $1050^{\circ} \mathrm{C}$ & $1.9 \times 10^{18}$ \\
\cline { 2 - 3 } & $1150^{\circ} \mathrm{C}$ & $1.6 \times 10^{18}$ \\
\cline { 2 - 3 } & $1250^{\circ} \mathrm{C}$ & $4.4 \times 10^{17}$ \\
\hline \hline
\end{tabular}



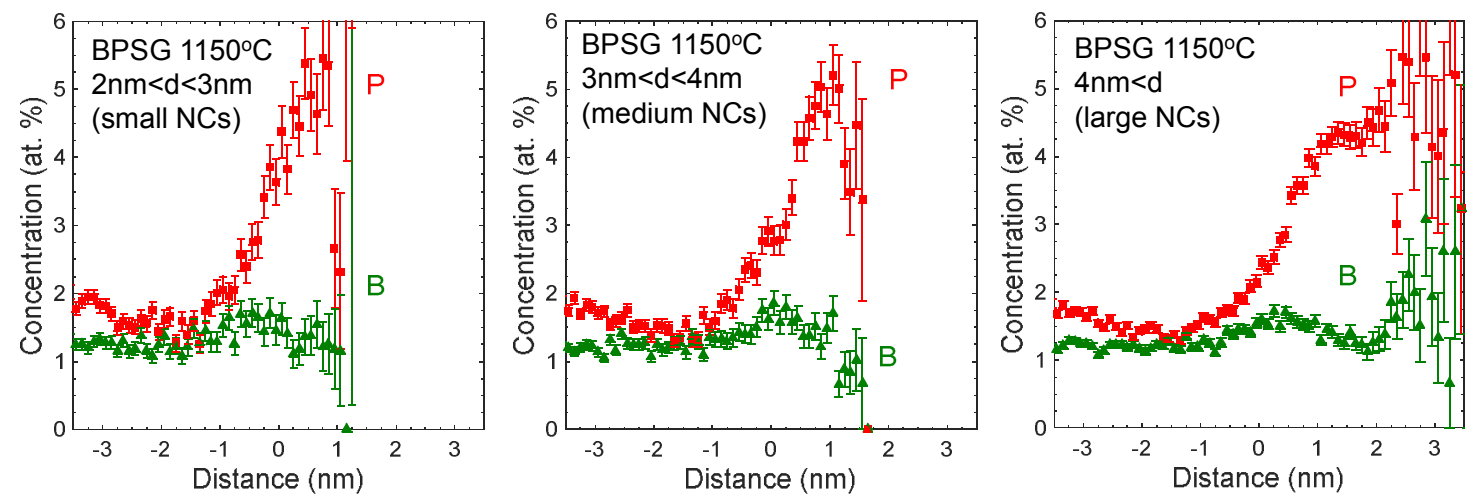

Figure S4. Proxigram analysis on co-doped Si NCs in BPSG annealed at $1150{ }^{\circ} \mathrm{C}$ for a variety of Si NCs size ranges. 
(a)

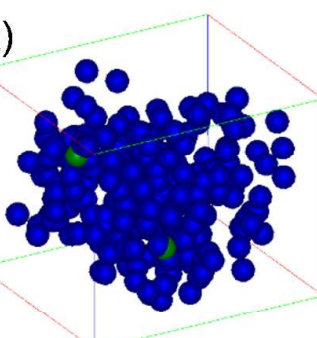

(b)

(c)
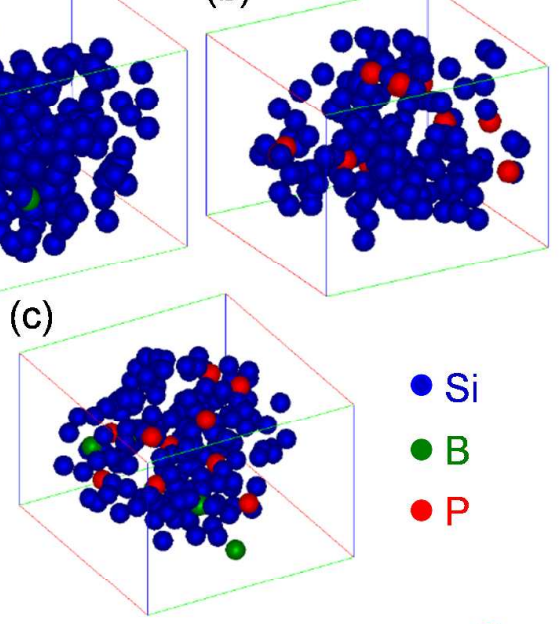

(d)

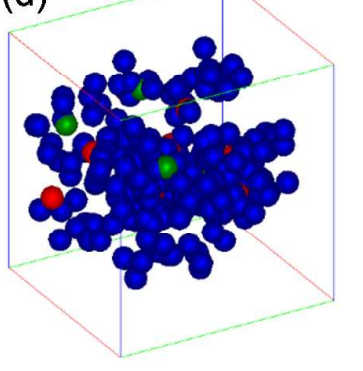

(f)

(e)
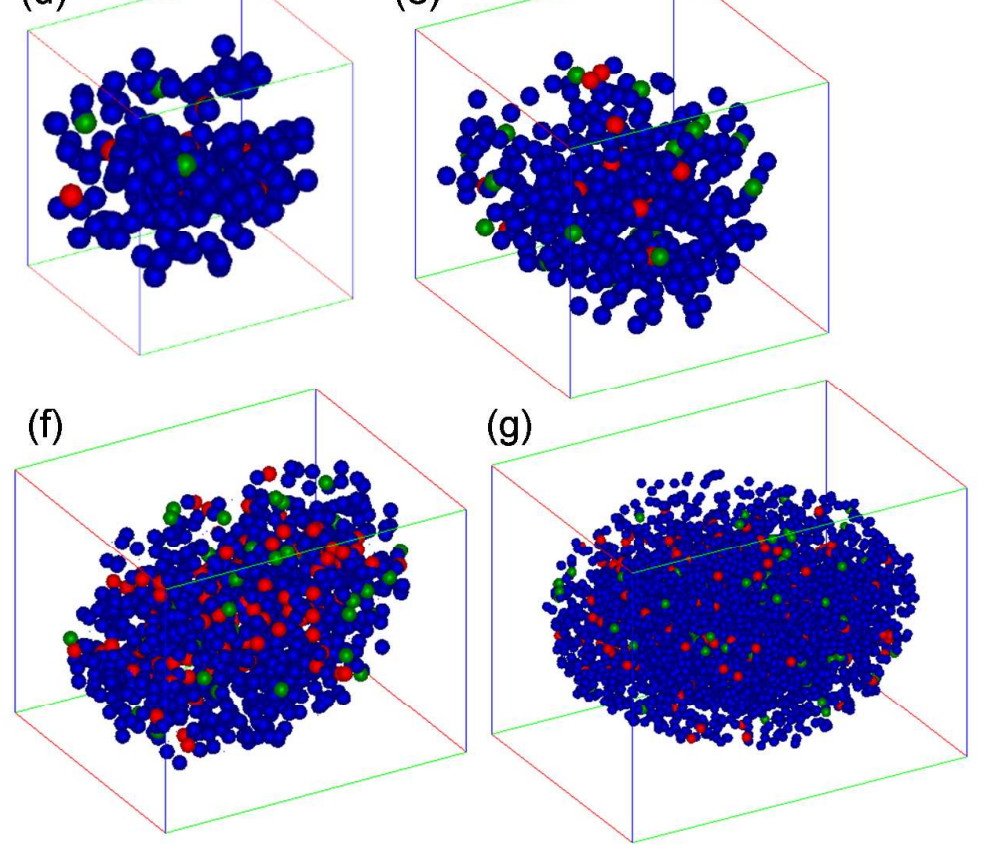

Figure S5. Individual Si NCs in (a) BSG annealed at $1150{ }^{\circ} \mathrm{C}$, (b) PSG annealed at $1150{ }^{\circ} \mathrm{C}$, (c) BPSG annealed at $1150{ }^{\circ} \mathrm{C}$, (d, e) BPSG annealed at $1050^{\circ} \mathrm{C}$, and (f, g) BPSG annealed at $1250{ }^{\circ} \mathrm{C}$. The diameter of each sample is (a-d) $2.0 \mathrm{~nm}$, (e) $3.0 \mathrm{~nm}$, (f) $3.9 \mathrm{~nm}$ and (g) $6.0 \mathrm{~nm}$. 